\title{
Control Global del Péndulo con Rueda de Reacción Empleado Redes Neuronales Artificiales y Linealización Extendida
}

\author{
Global Control of Reaction Wheel Pendulum Using Artificial Neural \\ Networks and Extended Linearization
}

\author{
Oscar Danilo Montoya Giraldo ${ }^{1}$, Carlos Alberto Ramírez Vanegas ${ }^{1}$, Luis Fernando Grisales \\ Noreña $^{2}$ \\ ${ }^{1}$ Ingeniera eléctrica, Universidad Tecnológica de Pereira, Pereira, Colombia. \\ ${ }^{2}$ Ingenieria eléctrica. Instituto Tecnológico Metropolitano de Medellín, Medellín Colombia \\ odmontoya@utp.edu.co \\ caramirez@utp.edu.co \\ luisgrisales@itm.edu.co
}

\begin{abstract}
Resumen- En este artículo se presenta el diseño y la simulación de un controlador híbrido de dos etapas para el péndulo invertido con rueda de reacción (RWP). En la primera fase se realiza el modelado general del brazo pendular a través de un modelo no lineal que determina la energía almacenada en la planta, y usando una estrategia de balance conocida como regulación de energía (RE), se obtienen los datos necesarios para el entrenamiento de una red neuronal artificial (RNA). En la segunda etapa, por medio de un sistema de conmutación suave, se intercambia el control neuronal por un controlador basado en la linealización extendida de las variables de estado ( $L E V E$ ), con lo cual se garantiza la permanencia del péndulo en la región de operación. La estrategia de control propuesta, muestra un excelente desempeño ante fenómenos de perturbación externa y garantiza la operatividad global del sistema físico.
\end{abstract}

Palabras clave- Control global, Conmutación suave, Linealización extendida, Modelos energéticos, Redes Neuronales Artificiales.

Abstract - In this paper describes the design and simulation of a two-stage hybrid controller for the inverted reaction wheel pendulum (RWP) is presented. In the first stage, the general pendulum arm modeling is performed through a nonlinear model to determine the stored energy in the plant, and using a strategy known as swing up energy regulation, the data required for the training of an artificial neural network are obtained. In the second stage, via a soft switching system, neuronal control is exchanged by a controller based on the extended linearization of the state variables, whereby the permanence of the pendulum in the region of operation is guaranteed. The control strategy proposed shows excellent performance against external disturbance phenomena and ensures the overall operation of the physical system.
Key Word - Global control, Soft commutation, Extended linearization, Energetic models, Artificial Neural Networks.

\section{ACRÓNIMOS}

SISO : : Entrada única - Salida única (Single Input Single Output).

RNA : Red neuronal artificial o su equivalente plural.

RE : Regulación de energía.

RWP : Péndulo invertido con rueda de reacción (Reaction wheel pendulum).

LEVE : Linealización extendida de las variables de estado.

\section{NOMENCLATURA}

\section{Parámetros y subíndices:}

$\begin{array}{lll}a, b, c & : & \begin{array}{l}\text { Constantes determinadas en función de los } \\ \text { parámetros físicos del RWP. }\end{array} \\ J_{P} & : \begin{array}{l}\text { Momento de inercia del péndulo, respecto del } \\ \text { punto de pivote. }\end{array} \\ J_{r} & : \begin{array}{l}\text { Momento de inercia de la rueda, respecto a su } \\ \text { centro de masa. }\end{array} \\ E_{S} & : \quad \begin{array}{l}\text { Energía almacenada en la barra pendular. } \\ g\end{array} & : \text { Constante de gravitación universal. } \\ m & : \text { Masa total del péndulo. } \\ l & : \begin{array}{l}\text { Longitud del péndulo, tomada desde el pivote } \\ \text { hasta su centro de gravedad. }\end{array}\end{array}$




\begin{tabular}{|c|c|c|}
\hline$K_{m}$ & & $\begin{array}{l}\text { Constante de relación entre el troque } \\
\text { aplicado por el motor y la señal de voltaje sus } \\
\text { terminales. }\end{array}$ \\
\hline$K_{u}$ & & $\begin{array}{l}\text { Constante de proporcionalidad para la señal } \\
\text { de control por regulación de energía. }\end{array}$ \\
\hline \multicolumn{3}{|l|}{ Variables: } \\
\hline$u$ & & control. \\
\hline$\varphi$ & & $\begin{array}{l}\text { ar del péndulo, respecto la } \\
\text { ilibrio (eje }+y) \text {. }\end{array}$ \\
\hline$\theta$ & & $\begin{array}{l}\text { lar absoluta de la rueda, con } \\
+y \text {. }\end{array}$ \\
\hline$\alpha$ & & $\begin{array}{l}\text { lar relativa de la rueda, con } \\
\text { pendular. }\end{array}$ \\
\hline$\tau$ & & icado por el motor DC a la rueda \\
\hline $\operatorname{sgn}(x)$ & & $\begin{array}{l}\text { Función que determina el signo de la variable } \\
\text { asociado a la variable } x \text {. }\end{array}$ \\
\hline
\end{tabular}

\section{INTRODUCCIÓN}

EL sistema físico RWP fue diseñado por Mark. W. Spong en 1995, con el fin de contribuir a la gama de péndulos invertidos disponibles, introduciendo variaciones sustanciales a los modelos existentes hasta aquel momento. Con lo cual se entregó a la comunidad académica un sistema físico que permite la aplicación de gran variedad de estrategias de control, tanto lineales, como no lineales. El sistema RWP hace parte del conjunto de péndulo invertidos, dentro de los que se destacan: el péndulo invertido clásico [1], el péndulo rotacional invertido o péndulo de Furuta [2] y el Pendubot [3] (péndulo conformado por dos brazos conectados en serie); que son ampliamente conocidos por su dinámica no lineal compleja, lo cual permite el análisis y diseño de controladores sofisticados que pueden operar en todo el rango de operación de la planta (controlador global), o diseñados para operar soló en regiones de operación especificas (controlador local).

Los sistemas pendulares, han sido analizados desde hace varias décadas, debido a que siempre presuponen un desafío notable para las técnicas de control clásicas como la realimentación de variables de estado [4], controladores PID [5], reguladores óptimos con criterio cuadrático [6] y planos deslizantes [7] entre otros; con lo que se da origen a modelos más complejos de control basado en técnicas metaheurísticas de optimización, tales como el recocido simulado [8], algoritmos genéticos [9], colonia de hormigas [10] y optimización por enjambre de partículas [11], entre otros. Por otro lado, también se destacan las redes neuronales artificiales [12], [13] y la lógica difusa [14], los cuales se basan en aprendizaje de comportamientos o descripciones aproximadas de la realidad, respectivamente.

Las condiciones de operación de los péndulos invertidos, han hechos que estos sistemas físicos, se hayan convertido en bancos de prueba altamente empleados para diseño, simulación y montaje de diversos esquemas de control, dada su complejidad matemática y dinámica no lineales, semejantes a sistemas de gran envergadura como los sistemas de comunicaciones, la aeronáutica, los sistemas de transporte, y en general con sistemas de uso industrial y militar como los puentes grúa y el lanzamiento de proyectiles [15].

Debido a las características anteriormente descritas, en este trabajo se selecciona el RWP, para realizar el diseño y la simulación de un controlador híbrido de dos etapas, integrando modelos de energía y redes neuronales artificiales para el levantamiento y acercamiento del péndulo a la región de operación, con un controlador local diseñado a través de un modelo de realimentación de variables de estado extendido, que pretende ampliar la región de operación local.

A diferencia de los trabajos anteriores, en este artículo presenta como aporte fundamental, la combinación de un controlador de balance, para llevar el péndulo a la posición vertical superior, a través del entrenamiento de una RNA, con datos obtenidos de un modelo clásico de RE y un controlador local diseñado por medio de la técnica robusta de control, conocida como LEVE. Adicionalmente, se presenta un esquema de conmutación suave que permite el intercambio de controladores por medio de una zona de operación nula (zona cero), con la cual se minimiza la aparición de pulsos indeseados en la señal de control.

\section{MODELOS MATEMÁTICOS CONSIDERADOS}

\section{A. Modelo matemático general para el RWP}

El RWP está conformado por una barra, que tiene acoplada en uno de sus extremos una rueda giratoria (rueda de reacción) conectada a motor DC y en su otro extremo conectada a un punto de pivote, que le da estabilidad mecánica. El par aplicado por el motor se usa para controlar el sistema, el cual está provisto de sensores de movimiento que entregan la posición relativa del ángulo para la rueda de reacción $(\alpha)$ y la posición angular de la barra $(\varphi)$. La Fig. 1 presenta una representación bidimensional del péndulo y las variables de interés.

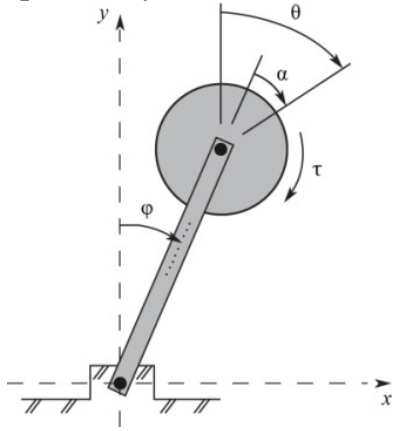

Figura 1. Modelo bidimensional del RWP

La relación entre el ángulo del péndulo y la medida absoluta del ángulo $(\theta)$ de la rueda resulta ser tal como se presenta en (1).

$$
\theta=\varphi+\alpha
$$

Para modelar este sistema físico, múltiples autores han propuesto diversas metodologías, dentro de las que se destacan 
la mecánica de LaGrange y los modelos de Newton [15], [16]. En este trabajo se toma como referencia, el modelo presentado en [2], el cual da origen a las expresiones (2) y (3).

$$
\begin{gathered}
J_{p} \ddot{\varphi}=m g l \sin (\varphi)-K_{m} u \\
J_{r} \ddot{\theta}=K_{m} u
\end{gathered}
$$

Con el fin de mostrar una representación simplificada del modelo descrito en (2) y (3), se introducen las constantes $a, b$ y $c$, que dependen de los parámetros físicos de la planta, con lo cual se llega (4) y (5).

$$
\begin{gathered}
\ddot{\varphi}=a \sin (\varphi)-b u \\
\ddot{\theta}=c u
\end{gathered}
$$

Los valores asociados a las constantes son tomados de la referencia [17], los cuales son: $a=78,4 ; b=1,08$ y $c=198$.

En la presentación de este modelo dinámico, se han despreciado los coeficientes de fricción entre el péndulo y el pivote y la rueda y el péndulo. Por otro lado, como la posición angular relativa de la rueda (ángulo $\theta$ ), es irrelevante en términos del control. Este modelo puede ser reducido, tomado como variables de interés, el ángulo del péndulo y su velocidad angular, y la velocidad angular de la rueda de reacción, es decir: $x_{1}=\varphi, x_{2}=\dot{\varphi}$ y $x_{3}=\dot{\theta}$. Al sustituir estas variables el modelo definido por (3) y (4), se encuentra una representación simplificada de orden tres, que representa al sistema físico RWP en términos de las variables de estado, tal como se muestra en (6).

$$
\begin{gathered}
\dot{x}_{1}=x_{2} \\
\dot{x}_{2}=a \sin \left(x_{1}\right)-b u \\
\dot{x}_{3}=c u
\end{gathered}
$$

El sistema en ecuaciones de estado presentado en (6), corresponden a un modelo no lineal que tiene la forma $\dot{x}=f(x, u)$ , donde $x$ corresponde al vector de estados de dimensión $3 x 1$ y $f(x, u)$ representa una función vectorial no lineal que asocia la dinámica del sistema con sus variables de estado. El modelo matemático descrito por (6) puede ser rescrito en forma matricial, como se muestra en (7).

$$
x=\left[\begin{array}{l}
x_{1} \\
x_{2} \\
x_{3}
\end{array}\right] \wedge f(x, u)=\left[\begin{array}{c}
x_{2} \\
a \sin \left(x_{1}\right)-b u \\
c u
\end{array}\right]
$$

Al analizar la dinámica no lineal del péndulo con rueda de reacción es claro, que es un sistema sub-actuado (dos grados de libertad y una única entrada de control). Por lo cual, las estrategias de control que se le aplica, tiene la finalidad de mantener la planta en sus puntos de equilibrio inestable y regular dichos estados alrededor de dichos puntos de operación [14]. Para determinar los puntos de equilibrio de la planta, basta con igualar la función vectorial no lineal que modela la dinámica de la planta a cero, considerando una entrada nula. Al realizar este análisis, es claro que: $\theta=k$ y $\varphi=2(n-1) \pi, n \in \square$, es decir, la posición vertical superior o posición invertida, que es inestable, y $\theta=k$ y $\varphi=(2 n-1) \pi, n \in \square$, que corresponde a la posición de equilibrio natural en la parte vertical inferior. Es de notar que la posición angular de la rueda de reacción no influye en la localización de los puntos de equilibrio ya que puede tomar cualquier valor real $k$. El objetivo fundamental de este trabajo, es llevar el péndulo desde su posición de equilibrio natural a la posición vertical superior y mantenerlo allí.

\section{B. Modelo matemático simplificado para RE}

Para aplicar el controlador por LEVE, será considera el modelo descrito en (7). Sin embargo, para realizar el entrenamiento de la RNA que realizará el balance del sistema, hasta alcanzar la posición vertical superior, se realiza un modelo conocido como regulación de los estados energéticos, que ha sido ampliamente utilizado en la literatura especializada [6], [12] y [16]-[24]. La idea básica del modelo de RE, es plantear un modelo que haga la energía total almacenada igual a cero en el punto de operación deseado y que se regule a través de la acción gradual de una señal de la aplicación de una señal de control que cumpla con este propósito. Para el sistema que se presentó en la Fig. 1, la relación entre la posición angular de la barra y la energía global de la planta corresponde a la presentada en (8).

$$
E_{s}=0.5 J_{P}(\dot{\varphi})^{2}+m g l(\cos (\varphi)-1)
$$

Es de notarse que se desprecia la energía cinética de la rueda de reacción $0.5 J_{r}(\dot{\theta})^{2}$, puesto que la velocidad angular de la rueda no incide en la posición angular de la barra. Tomando la derivada temporal de la energía, se obtiene la expresión presentada en (9).

$$
\dot{E}_{s}=\dot{\varphi}\left(J_{P} \ddot{\varphi}-m g l \sin (\varphi)\right)
$$

Por comparación con el modelo global de la planta presentado en (2), es claro que resulta ser (10).

$$
\dot{E}_{s}=-\dot{\varphi} K_{m} u
$$

Analizando la expresión para la energía del péndulo presentada en (8), es claro que su valor mínimo (valor negativo, según la convención asignada), se encuentra cuando el péndulo se encuentra en su posición de equilibrio natural (posición vertical inferior) y se hace nula, cuando este alcanza la posición de equilibrio inestable en la posición vertical superior. Para garantizar que la señal de control, lleve la función de energía a cero, es necesario que $\dot{E}_{s}>0$, por lo tanto, al observar la expresión (10), la condición de positividad se cumple si el producto $\dot{\varphi} u$ es negativo. Aunque en la literatura especializada, es posible encontrar diferentes formulaciones para representar la función de control, la expresión más destacada es la que se 
muestra en (11), la cual emplea la energía del sistema y el signo de la velocidad angular del péndulo.

$$
u=K_{u} E_{s} \operatorname{sgn}(\dot{\varphi})
$$

El objetivo de la función $\operatorname{sgn}(x)$ en la expresión (11) permite que la señal de control refuerce el movimiento del péndulo, es decir, que el torque aplicado por el motor, es tal que tiene la misma dirección del movimiento de la barra pendular. La constante $K_{u}$ permite graduar la amplitud de la señal reguladora y la rapidez con la que el péndulo alcanza la región de operación; esta constante tiene un ajuste empírico y depende las características dinámicas de la planta.

\section{MÉTODOS DE LINEALIZACIÓN APLICABLES AL RWP}

El modelo matemático empleado en el desarrollo de esta sección ha sido tomado de la referencia [21], en la cual los autores muestran la deducción del modelo linealizado para el péndulo con rueda de reacción.

\section{A. Método de linealización aproximada}

Una representación general del modelo de estados de un sistema no lineal de orden $n$ tiene asociadas las ecuaciones (14) y (15).

$$
\begin{gathered}
\dot{x}=f(x, u) \\
y=g(x)
\end{gathered}
$$

Cuando se analiza un sistema del tipo SISO, $x$ es un vector de $R^{n}$, $f(x, u)$ es una función vectorial suave $\left(f: R^{n+1} \rightarrow R^{n}\right)$ y $g(x)$ es una función escalar suave $\left(g: R^{n} \rightarrow R\right)$. Para usar estrategias de control lineal alrededor de un punto de operación $x_{0}$ dado, se obtiene un modelo linealizado considerando los términos de primer orden de la expansión en serie de Taylor, es decir, las expresiones (14) y (15).

$$
\begin{gathered}
\Delta \dot{x}=\left.\frac{\delta f}{\delta x}\right|_{x_{0}} * \Delta x+\left.\frac{\delta f}{\delta u}\right|_{x_{0}} * \Delta u \\
\Delta y=\left.\frac{\delta g}{\delta x}\right|_{x_{0}} * \Delta x
\end{gathered}
$$

Al evaluar las derivadas en el punto de operación se obtienen las matrices del modelo linealizado alrededor del punto de operación se obtiene entonces el modelo de estados lineal dado por (16).

$$
\begin{gathered}
A=\delta f /\left.\delta x\right|_{x_{0}} ; B=\delta f /\left.\delta u\right|_{x_{0}} ; C=\delta g /\left.\delta x\right|_{x_{0}} \\
\Delta \dot{x}=A \Delta x+B \Delta u \\
\Delta y=C \Delta x
\end{gathered}
$$

En el cual $\Delta x, \Delta u$ y $\Delta y$ representan las variaciones de los estados, la entrada y la salida respectivamente alrededor del punto de operación definido en forma general como
$\Delta x=x-x_{0} ; \Delta u=u-u_{0} \wedge \Delta y=y-y_{0}$, Sobre el modelo linealizado se puede diseñar un regulador lineal de la forma $\Delta u=-K \Delta x$ (siempre que el par $(A, B)$ sea controlable). Las ganancias de realimentación $K=\left[K_{1} K_{2} \ldots K_{n}\right]$ se calculan de forma que en lazo cerrado se obtengan los polos o valores propios deseados [21].

\section{B. Método de linealización extendida}

Si en el modelo linealizado del sistema no se especifica un punto de operación dado, se obtienen expresiones genéricas en función de los estados del punto de operación $x_{0}=\left[\begin{array}{llll}x_{10} & x_{20} \ldots & x_{n 0}\end{array}\right]^{T}$. Al diseñar un regulador, las ganancias $\bar{K}$ quedan también definidas en términos de $x_{0}$, lo que permite proponer una ley de control no lineal de la forma (17).

$$
u=-K(x)
$$

Que sea una extensión del regulador lineal alrededor de $x_{0} \mathrm{y}$ cuya linealización corresponde a la expresión (28).

$$
u=-\left.\frac{\delta K}{\delta x_{1}}\right|_{x_{0}} \Delta x_{1}-\left.\frac{\delta K}{\delta x_{2}}\right|_{x_{0}} \Delta x_{2}-\cdots-\left.\frac{\delta K}{\delta x_{n}}\right|_{x_{0}} \Delta x_{n}
$$

Si $\bar{K}=\left[\bar{K}_{1} \bar{K}_{2} \ldots \bar{K}_{n}\right]$ es el regulador lineal clásico alrededor del punto de operación $(\Delta u=-K \Delta x)$, se impone el conjunto de condiciones (19) sobre la función no lineal $K(x)$ con el fin de que ambos controladores coincidan en el punto $x_{0}$.

$$
\left.\frac{\delta K}{\delta x_{i}}\right|_{x_{0}}=\bar{K}_{i} ; i=1,2, \ldots, n
$$

Si no se ha especificado un punto de operación numérico, es posible hallar una expresión para $K(x)$ integrando estas condiciones con respecto a las variables $x_{10} x_{20} \ldots x_{n 0}$. Aunque este problema puede tener múltiples soluciones, se puede obtener un resultado sencillo si $K(x)$ se define de una forma conveniente [21].

\section{Método de linealización extendida aplicado al RWP}

Por ser un sistema sub-actuado, el péndulo con rueda de reacción solo se puede controlar en las posiciones de equilibrio (la posición natural $\phi= \pm \pi, \pm 3 \pi, \pm 5 \pi, \ldots$ o la invertida $\phi=0, \pm 2 \pi, \pm 4 \pi, \ldots)$. Aunque inicialmente se considera un punto de operación arbitrario en el cálculo del regulador no lineal $u=-K(x)$, posteriormente se escogerá la posición invertida para realizar las simulaciones. 
Si el conjunto de ecuaciones (6) que describe la dinámica del modelo se linealiza alrededor de un punto de operación $x_{0}$ usando (14) y (15) se obtienen 20) y (21).

$$
\begin{gathered}
{\left[\begin{array}{c}
\Delta \dot{x}_{1} \\
\Delta \dot{x}_{2} \\
\Delta \dot{x}_{3}
\end{array}\right]=\left[\begin{array}{ccc}
0 & 1 & 0 \\
a \cos x_{1} & 0 & 0 \\
0 & 0 & 0
\end{array}\right]\left[\begin{array}{l}
\Delta x_{1} \\
\Delta x_{2} \\
\Delta x_{3}
\end{array}\right]+\left[\begin{array}{c}
0 \\
-b \\
c
\end{array}\right] \Delta u} \\
\Delta y=\left[\begin{array}{lll}
1 & 0 & 0
\end{array}\right]\left[\begin{array}{l}
\Delta x_{1} \\
\Delta x_{2} \\
\Delta x_{3}
\end{array}\right]
\end{gathered}
$$

Donde se pueden identificar las matrices $A, B$ y $C$ mostradas en (16). La matriz de controlabilidad asociada a este modelo se define como (22).

$$
W_{c}=\left[\begin{array}{lll}
A & A B & A^{2} B
\end{array}\right]=\left[\begin{array}{ccc}
0 & -b & 0 \\
-b & 0 & -a b \cos x_{1} \\
c & 0 & 0
\end{array}\right]
$$

Y cuyo determinante es $\operatorname{det}\left(W_{c}\right)=a b^{2} c \cos x_{10}$, lo que implica que el sistema es controlable ( $W_{c}$ tiene rango tres), excepto en los puntos de operación $\phi= \pm \pi / 2$ o equivalentes. Se plantea una señal de control como se indica en (17) $u=-K(x)$ con la definición presentada en (23) y se impone la condición (24).

$$
\begin{gathered}
u=-K_{1}(x)-K_{2}(x)-K_{3}(x) \\
\left.\frac{\delta K}{\delta x_{i}}\right|_{x_{0}}=\bar{K}_{i} ; i=1,2,3
\end{gathered}
$$

Siendo $\bar{K}=\left[\begin{array}{lll}\bar{K}_{1} & \bar{K}_{2} & \bar{K}_{3}\end{array}\right]$ la matriz de ganancias del regulador lineal en la posición invertida $\phi=0$ (y sus equivalentes). Este último se obtiene asignando valores propios adecuados a la matriz de lazo cerrado $A_{L C}=A-B \bar{K}$, es decir, como (25).

$$
|s I-(A-B \bar{K})|=\left(s+\lambda_{1}\right)\left(s+\lambda_{2}\right)\left(s+\lambda_{3}\right)
$$

$\mathrm{Al}$ realizar las operaciones indicadas en (24) usando las matrices de (20) y (21) y el vector $\bar{K}$ se obtienen los resultados (26), (27) y (28).

$$
\begin{gathered}
\bar{K}_{1}(x)=-\frac{\beta+a \cos x_{10}}{b}=\frac{\delta K_{1}}{\delta x_{10}} \\
\bar{K}_{2}(x)=-\frac{1}{b}\left(\frac{\gamma}{a} \sec x_{10}+\alpha\right)=\frac{\delta K_{2}}{\delta x_{20}} \\
\bar{K}_{3}(x)=-\frac{\gamma}{a c} \sec x_{10}=\frac{\delta K_{3}}{\delta x_{30}}
\end{gathered}
$$

Las constantes $\alpha, \beta$ y $\gamma$ se relacionan con los valores propios deseados $\left(\lambda_{1}, \lambda_{2}, \lambda_{3}\right)$ mediante las expresiones $\alpha=\lambda_{1}+\lambda_{2}+\lambda_{3}, \beta$ $=\lambda_{1} \lambda_{2}+\lambda_{1} \lambda_{3}+\lambda_{2} \lambda_{3}, \mathrm{y} \gamma=\lambda_{1} \lambda_{2} \lambda_{3}$.

De las ecuaciones (26), (27) y (28) se obtienen las funciones
$K_{l}(x), K_{2}(x)$ y $K_{3}(x)$ si se integra en cada una con respecto a variables que sustituyen a los valores de punto de operación, respectivamente $x_{10}, x_{20}$ y $x_{30}$. Los resultados se muestran en las ecuaciones (29), (30) y (31).

$$
\begin{aligned}
& \bar{K}_{1}(x)=-\int_{x_{10}}^{x_{1}} \frac{\beta+a \cos v}{b} d v=\frac{\beta\left(x_{10}-x_{1}\right)+a\left(\sin x_{10}-\sin x_{1}\right)}{b} \\
& \bar{K}_{2}(x)=-\int_{x_{20}}^{x_{2}} \frac{1}{b}\left(\frac{\gamma}{a} \sec x_{10}+\alpha\right) d v=\frac{1}{b}\left(\frac{\gamma}{a} \sec x_{1}+\alpha\right)\left(x_{20}-x_{2}\right) \\
& \bar{K}_{3}(x)=-\int_{x_{30}}^{x_{3}} \frac{\gamma}{a c} \sec x_{10} d v=\frac{\gamma}{a c}\left(x_{30}-x_{3}\right) \sec x_{1}
\end{aligned}
$$

Al sustituir (29), (30) y (31) en (22) se obtiene el regulador no lineal que extiende a su equivalente linealizado $(\Delta u=-\bar{K} \Delta x)$ alrededor del punto de operación $x_{10}=0$.

\section{REDES NEURONALES ARTIFICIALES}

El aprendizaje de los seres inteligentes está caracterizado por el desarrollo de secuencial de las células neuronales, desde el momento mismo de su nacimiento, y su aprendizaje se da en base a la experiencia y al conocimiento del entorno en el que los seres vivos se desenvuelven, guiando su comportamiento hasta alcanzar un alto grado de independencia, lo que permite tomar decisiones para aquellas situaciones que no se habían presentado durante la etapa de aprendizaje. Es así como las redes neuronales biológicas son el fundamento de las redes neuronales artificiales, en donde cada uno de los componentes naturales es representado mediante su equivalente artificial con el fin de emular su comportamiento y obtener así complejos sistemas computacionales capaces de desempeñarse en forma correcta ante diferentes escenarios [12].

Las redes neuronales biológicas constan de tres elementos o componentes esenciales para su formación e interacción; en primer lugar, las dendritas son las encargadas de la recepción de las señales eléctricas provenientes de células adyacentes y trasportarlas al soma o cuerpo de la célula; el soma es el componente central de la neurona y está encargado de procesar la información y dar una señal salida que es entrega por el axón a las dendritas de las células vecinas. Con base en la composición natural de las redes neuronales naturales, es posible plantear una red neuronal artificial capaz de recibir información, procesarla y dar una señal de salida. Realizando una analogía del comportamiento biológico una RNA está conformada por un vector $p_{i}$ de entradas el cual representa las señales eléctricas provenientes de las células adyacentes y que son recibidas por las dendritas, en segundo lugar consta de una matriz de pesos $W_{i}$ que representa la intensidad de la conexión o sinapsis y por último de una función suma que es el operador de activación que permite la activación o no de la célula y permitir así una señal de salida, este proceso se da en el soma. La Fig. 2 presenta una red neuronal biológica (a) y su equivalente artificial (b). Donde $b$ representa una ganancia escalar que refuerza la salida del sumador $n$, el cual se constituye como la salida neta de la neurona y es pasado a través de una función de transferencia $f$ que puede ser de 
características lineales o no lineales en función de $n$ y su escogencia depende del tipo de problema que sea analizado.

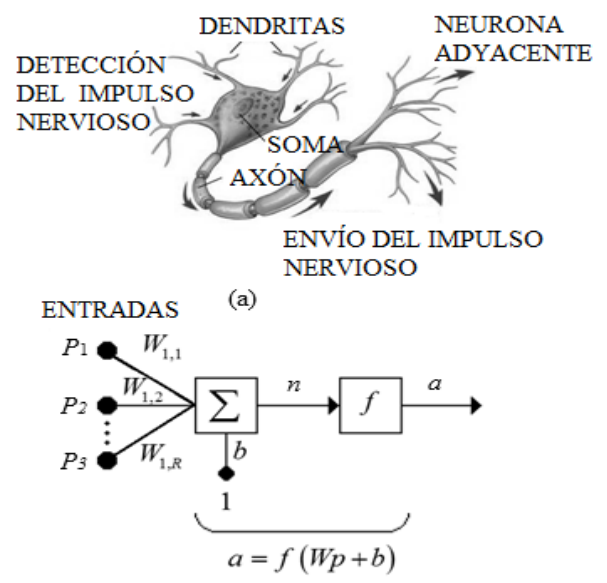

(b)

Figura 2. En (a) se muestra las características físicas de una neurona biológica y (b) muestra su extrapolación al mundo matemático.

Donde $b$ representa una ganancia escalar que refuerza la salida del sumador $n$, el cual se constituye como la salida neta de la neurona y es pasado a través de una función de transferencia $f$ que puede ser de características lineales o no lineales en función de $n$ y su escogencia depende del tipo de problema que sea analizado.

\section{A. Redes neuronales artificiales multicapa}

Como las redes neuronales biológicas son complejas y constan de millones de células interconectadas, es posible crear una red neuronal artificial multicapa capaz de procesar grandes bloques de información y entregar múltiples señales de salida que sean adaptables a diferentes entornos de trabajo. Este tipo de redes, son basadas en las posibles conexiones entre diferentes neuronas en una misma capa, es decir, que una capa puede estar constituida por $s$ neuronas, cada una de ellas encargada de recibir información y procesarla, dando como señal de salida una señal eléctrica que puede ser o no de activación que se entrega como resultado del procesamiento del conjunto de entrada. La Fig. 3 ilustra una red neuronal artificial multicapa (3 capas), en donde se observa la capa de entrada, una capa oculta y la capa de salida.

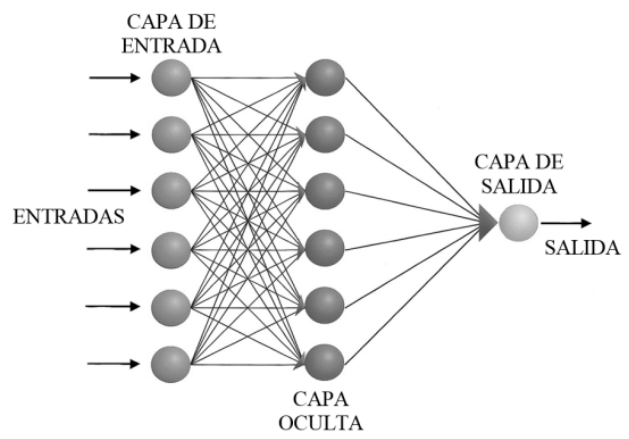

Figura 3. Configuración clásica de una RNA multicapa
Para lograr una red multicapa es necesario interconectar en forma secuencial un conjunto de capas con la posibilidad de tener o no un diferente número de neuronas, para la cual existirá como mínimo una capa de entrada y una capa de salida, que puede ser inclusive la capa de entrada, en el caso de una red de una sola capa.

\section{a. Red Neuronal Backpropagation}

Esta red neuronal artificial es un tipo de red que opera bajo un esquema de aprendizaje supervisado, es decir, que debe conocer la salida que desea obtener. Una forma adecuada de entender la de una red neuronal multicapa de esta naturaleza, es que para un conjunto específico de entrada (datos de entrenamiento), la señal de salida se propaga por cada una de las capas hasta la capa de salida generando así una señal de salida que es comparada con la señal que se deseada, con lo que se obtiene un error totalizado. Una vez obtenido este error se prepaga desde la capa final hasta la inicial, indicando así la contribución de cada neurona a la señal de error total [25], [26].

Este proceso de evolución de la señal de salida y devolución de la señal de error, permite durante la etapa de entrenamiento que la red neuronal identifique atributos y componentes especiales presentes en el patrón de entrada, que hacen parte del conocimiento adquirido y posibilita la capacidad de tomar decisiones adecuadas ante situaciones no presentadas en la etapa de entrenamiento, con base en atributos semejantes al patrón de entrenamiento original. La estructura de la red neuronal depende exclusivamente de los criterios del diseñador; sin embargo, una topología inicial de red puede considerar como cantidad de neuronas en la capa de entrada en número de señales de entrada, la capas ocultas tienen la libertad de considerar cantidades diferentes de neuronas y están más relacionadas con la experiencia del diseñador, finalmente el número de neuronas en la capa de salida se relaciona directamente con el número de señales de salida que se desea obtener.

\section{B. Modelo de aprendizaje inverso}

Una forma clásica de entrenar redes neuronales artificiales [12], [21], es usar el modelo de aprendizaje inverso, como se observa en la Fig. 4.

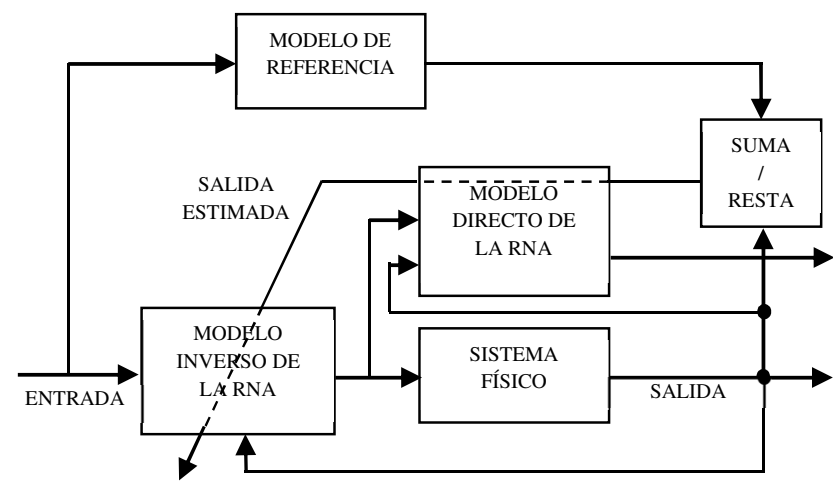

Figura 4. Esquema de aprendizaje supervisado empleado el modelo inverso 
En este modelo, la red neuronal usa un modelo que permita obtener la información necesaria para su entrenamiento y en caso del control de sistemas no lineales, consiste en la señal de entrada (señal de control) que corresponde la señal de salida que se desea obtener y el comportamiento de las variables de estado del sistema (señales de entrada). Una vez se tiene el conjunto de datos de entrada y salida se procede a entrenar la red usando un algoritmo de aprendizaje adecuado. El entrenamiento de la RNA termina cuando la diferencia entre la señal de salida de la red y la señal de salida original no supere el límite de convergencia preestablecido que asegura que no exista sobre-entrenamiento de la red, es decir, funcionamiento ideal para el conjunto de entrada, pero señales indeseadas ante variaciones en conjunto de entrada. En este contexto el límite de convergencia busca preservar la capacidad de generalización de las redes neuronales artificiales biológicas [12], [21].

\section{INTEGRACIÓN DE LOS CONTROLADORES}

Para realizar el diseño del controlador global para el RWP, en la sección III c, denominada modelo matemática para $R E$, se presentó el modelo matemático que permite al péndulo alcanzar la posición vertical superior, desde su posición de equilibrio natural, por medio de la variación energética de la planta. Al realizar la estrategia de balance por RE, se recolectan los datos necesarios para el entrenamiento de la RNA propuesta. Los datos de entrada en el proceso de entrenamiento serán la posición y velocidad angular del péndulo y como señal objetivo se considera el valor de la señal de control $u$ dada en (11).

Una vez el péndulo ha alcanzado la posición de equilibrio inestable en la posición vertical superior, el controlador mediante RNA es conmutado hacia el controlador por LEVE, el cual está encargado de mantener el péndulo en dicha posición, regulando el valor de todas las variables de estado, tal como se observa en la Fig. 5.

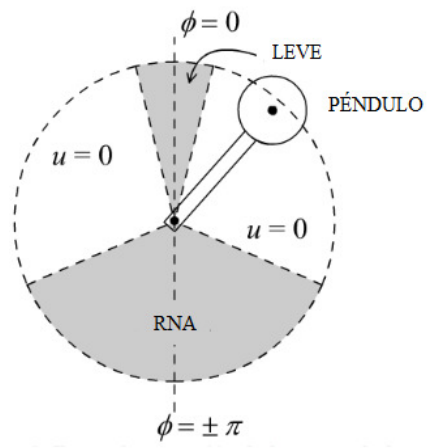

Figura 5. Región de operación de las señales de control

Con el fin de reducir el ruido eléctrico en las señales de control en el momento que se produce la conmutación, se realiza un proceso de transición suave entre los controladores, por medio de una función de control a trozos como la que se muestra en (32).

$$
u=\left\{\begin{array}{c}
K_{u} E_{s} \operatorname{sgn}(\dot{\varphi}) \rightarrow \mathrm{RNA} \\
0 \\
-K(x) \rightarrow \mathrm{LEVE}
\end{array}\right.
$$

\section{APLICACIÓN Y RESULTADOS}

El diseño y la simulación del controlador global para el péndulo con rueda de reacción empleando RNA y REVE se realizó en el software MATLAB 2014a en un computador Intel Core i3, con sistema operativo Windows 7 Professional y 4GB RAM.

\section{A. Entrenamiento de la RNA}

Se empleó el comando newff para crear una red neuronal del tipo backpropagation por medio de la utilización del tolbox de redes neuronales artificiales disponible para Matlab 2014a. Este comando requiere de la información básica para el entrenamiento, que corresponde a los datos de entrada, la salida deseada, el número de capas y la función de transferencia de cada capa, así como el algoritmo de entrenamiento.

Para el caso del péndulo con rueda de reacción, las entradas son tres: $p_{1}=\varphi$ y $p_{2}=\dot{\varphi}$, que corresponden a posición y velocidad del péndulo, respectivamente. La salida se toma como la señal objetivo del entrenamiento y corresponde a la señal RE (en la Fig. 6 se presenta la estructura del controlador por RE implementado en el simulink para entrenar la RNA).

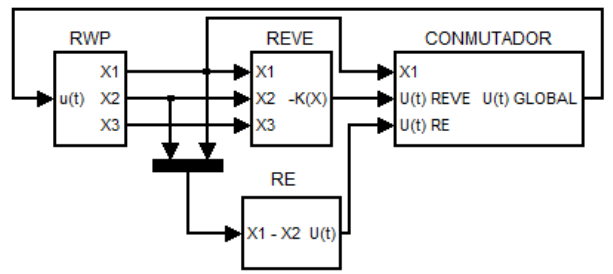

Figura 6. Estructura del controlador empleado RE y REVE

Por otro lado, se selecciona como configuración óptima de la red, una topología con tres capas, la primera con 2 neuronas (una por cada entrada), la segunda con 8 neuronas en la capa oculta y la tercera con 1 neurona en la capa de salida. Las funciones de transferencia para cada una de las capas es tansig, tansig y purelin Los parámetros de control de la RNA, como el caso de los pesos de la matriz de conexión de capas $W$ y las señales de activación $b$ de las neuronas, son ajustadas de manera interna por el algoritmo de entrenamiento (para este modelo se empleó el algoritmo Levenberg-Marquardt (trainlm en Matlab)) y no requieren de inicialización. En la Fig. 7 se muestra la topología de la red neuronal implementada.

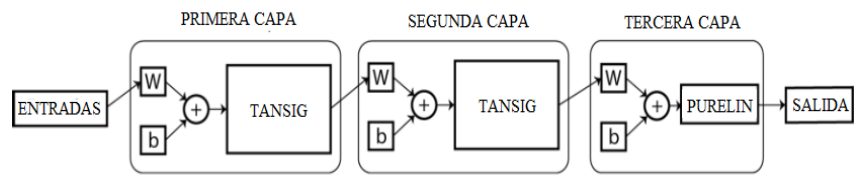

Figura 7. Configuración de la RNA implementada 
En la Fig. 8 se muestran las señales de entrada que alimentan la RNA y que corresponden a la velocidad y posición angular de la barra pendular y en la Fig. 9 la comparación entre la señal obtenida por RE y la señal de salida de la RNA.

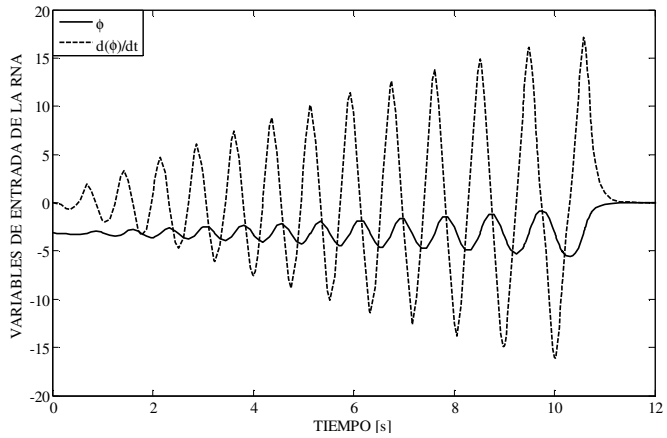

Figura 8. Señales de entrada para el entrenamiento de la RNA

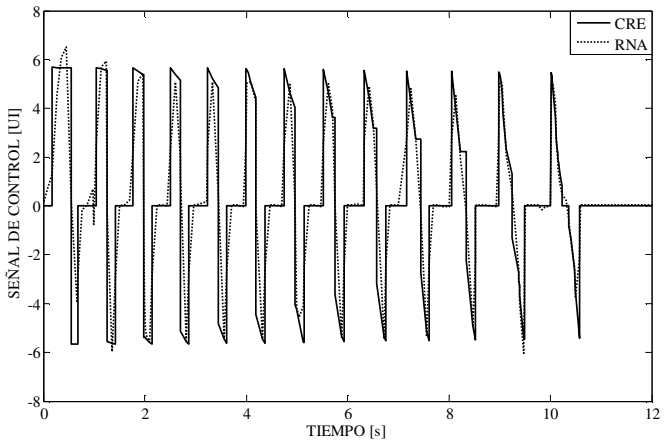

Figura 9. Comparación entre la señal obtenida por la implementación de RE y la RNA

Es de notarse (ver Fig. 9) que la salida de la red difiere de la obtenida por RE, y esto se debe a la capacidad que tienen las RNA para generalizar comportamientos y no para memorizarlos, lo que implica capacidad de operación ante componentes en las señales de entrada que no le fueron presentadas en el conjunto de entrenamiento original.

Una vez se ha obtenido la RNA, en el diagrama de bloques que se presentó en la Fig. 6 se cambia el bloque RE por la RNA, con lo que se tiene el nuevo diagrama de bloques que se muestra en la Fig. 10.

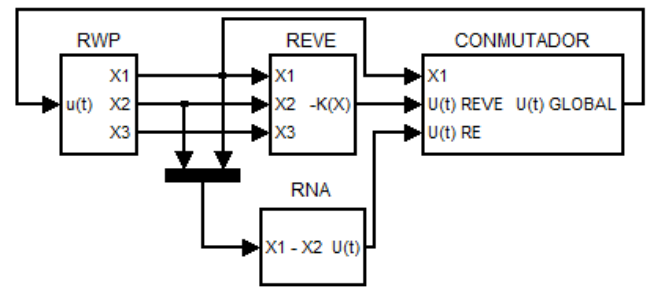

Figura 10. Estructura del control global con RNA y REVE

B. Pruebas de validación de la estrategia de control global propuesta

Para verificar la eficiencia en el diseño del controlador propuesto, se realizan dos escenarios de simulación clásicos al modelo matemático del RWP. El primer escenario de simulación consiste en introducir al sensor de posición angular del péndulo, que emulan el comportamiento real de los sensores por falta de calibración y desgaste por tiempo de uso; en el segundo escenario se realizan variaciones a la señal de control, que simulan el comportamientos de ruido, producidos por variaciones electromagnéticas y desgaste de la vida útil del motor DC, que se conoce como zona muerta, en la que los efectos de fricción impiden el movimiento del eje del motor (torque) para ciertos valores de la señal de entrada.

\section{1) Primer escenario: perturbaciones en el sensor de posición angular del péndulo}

En los lugares de operación de los sistemas de control, es común encontrar ambientes hostiles de trabajo, que deterioran rápidamente la vida útil de sensores y equipos de medición. Por esta razón, como los sensores, pueden sufrir desgaste y perdida de calibración, es necesario en ambientes simulados, realizar pruebas que permitan verificar el comportamiento del controlador ante este tipo de condiciones de operación. En la Fig. 11. Se muestra una señal de ruido aleatorio presente en el sensor de posición que emula un error en la medición de $\pm 1^{\circ}$.

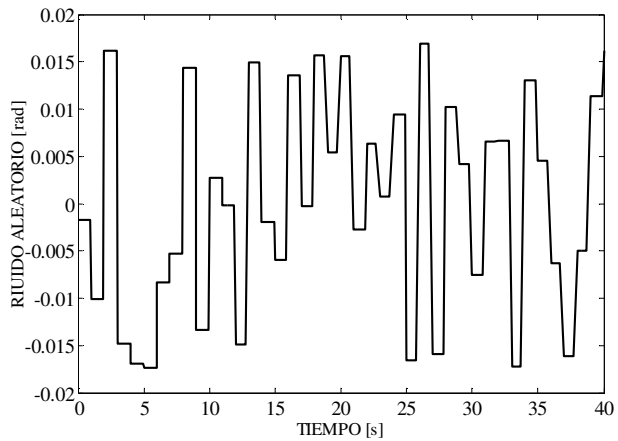

Figura 11. Ruido en la medición de la posición angular del péndulo

La variación en la medición de la posición angular del péndulo, implica movimientos suaves del péndulo alrededor del punto de operación, como se muestra en la Fig. 12, lo que genera un fenómeno conocido en particularmente en electrónica, como rizado en la señal de salida y corresponde a pequeñas diferencias entre la salida ideal y la salida real.

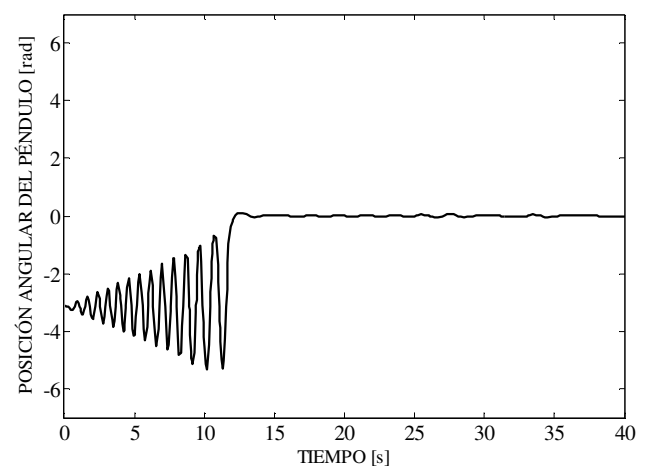

Figura 12. Comportamiento de la posición angular del péndulo ante una operación con ruido en su sensor 
Para mantener el péndulo en su región de operación, en la señal de control global que se presenta en la Fig. 13, es claro que las perturbaciones en la posición angular no inciden drásticamente en la etapa de balance con la RNA; sin embargo, cuando se alcanza la posición de equilibrio, es de notarse que la señal tiene variaciones no uniformes alrededor de cero (0) y con dirección contraria al efecto de perturbación en el sensor. Lo cual muestra que el control por REVE es muy eficiente a la hora de mantener el péndulo en la posición vertical invertida o región de operación inestable.

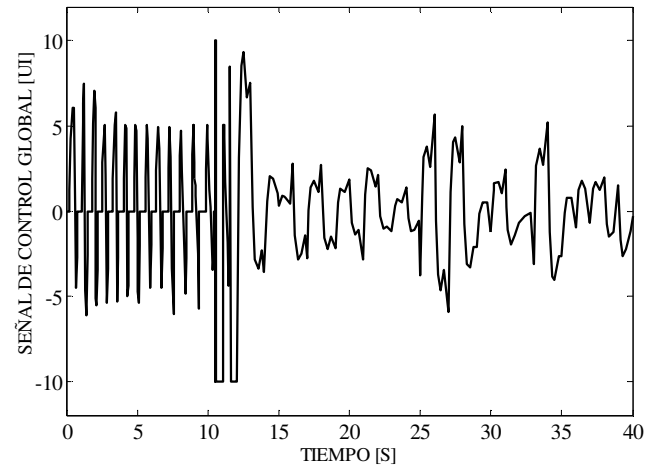

Figura 13. Señal de control global para una operación con ruido en el sensor de posición angular del péndulo

Para observar el efecto de perturbaciones externas a la posición angular del péndulo, se presenta una de perturbación externa que modifica la posición de la barra pendular con una magnitud que varía entre $-4^{\circ}$ y $3^{\circ}$ y emulan alteraciones que pueden sacar al RWP de la posición vertical invertida (ver Fig. 14), con lo que se busca mostrar como la RNA tiene la capacidad de reintegrar de manera muy rápida el péndulo a dicha posición de operación.

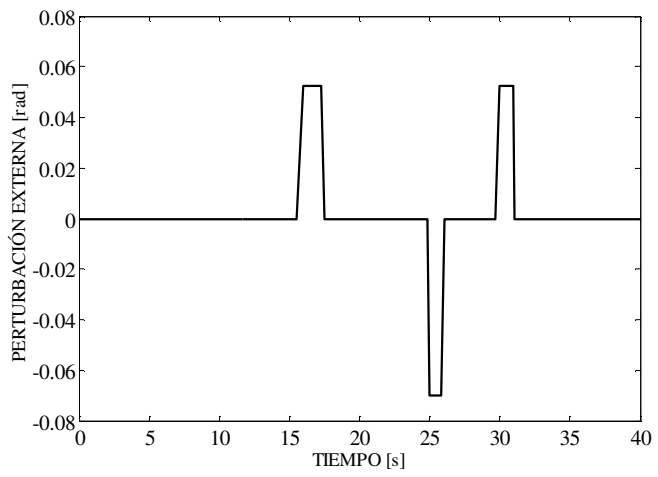

Figura 14. Comportamiento de la posición angular del péndulo ante una operación con ruido en su sensor

Por efecto de las perturbaciones externas que afectan la posición angular del péndulo en la Fig. 15 se muestra el comportamiento de la barra pendular en todo su rango de operación.

Cuando aparece la primera perturbación externa de $3^{\circ}$ a los $16 \mathrm{~s}$ la posición del péndulo tiene una variación suave alrededor de la zona de equilibrio; sin embargo, cuando aparece una perturbación de $-4^{\circ}$ a los $25 \mathrm{~s}$, el péndulo es sacado de su posición de equilibrio, con lo cual la RNA actúa y lo impulsa a llegar nuevamente a la posición de equilibrio en los $2 \pi \mathrm{rad}$ $\left(360^{\circ}\right)$, con respecto al eje de referencia (ver Fig. 16.).

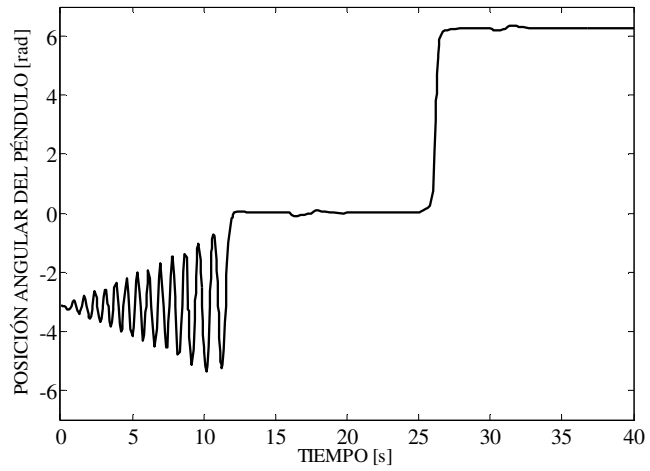

Figura 15. Comportamiento de la posición angular del péndulo ante una operación con perturbaciones externas

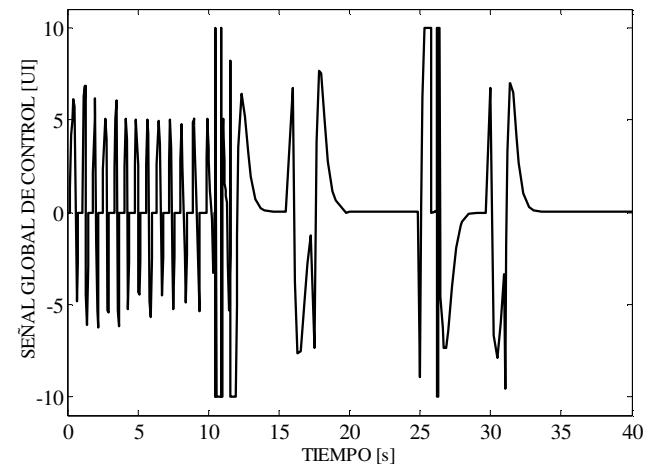

Figura 16. Señal de control global para una operación perturbaciones externas en la posición angular del péndulo

Finalmente, cuando aparece de nuevo una perturbación de $3^{\circ}$ a los 30 s se presenta nuevamente una variación suave del péndulo alrededor del punto de operación y el controlador REVE logra mantenerlo en dicha posición.

\section{2) Tercer escenario: perturbaciones en la señal de control}

Las señales de control, normalmente corresponden a señales eléctricas (voltaje o corriente) que alimentan actuadores que son típicamente motores (motor DC para el RWP). Debido a fenómenos electromagnéticos producidos en los ambientes de trabajo de los sistemas físicos, que son causados por señales eléctricas de potencia, estas perturbaciones pueden afectar su operación, generando valores no deseados en la entrada de los sistemas de control. Para emular este comportamiento en simulación, en la Fig. 17 se propone una alteración en la señal de control que emula la desconexión del controlador por un periodo de $1.5 \mathrm{~s}$ y que inicia a los $11 \mathrm{~s}$, además de un pulso que varía drásticamente la magnitud de la salida a los $25 \mathrm{~s}$ y con una duración de $0.5 \mathrm{~s}$ y una magnitud de -5UI, que emula interferencias electromagnéticas producidas por cortocircuitos o eventos de descargas atmosféricas cercanas a la posición geográfica del controlador. 


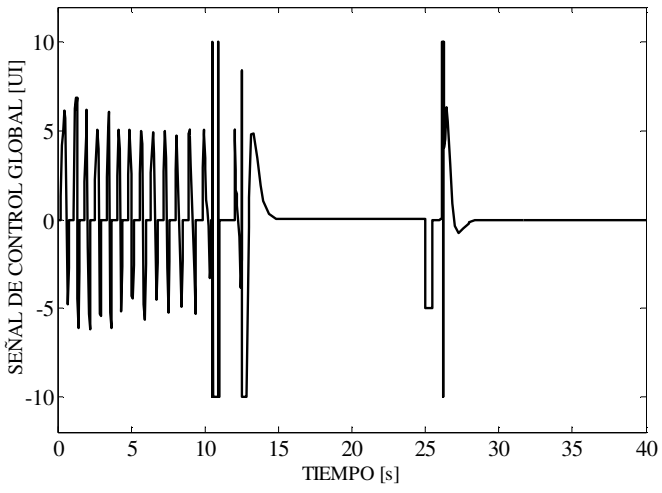

Figura 17. Señal de control global en presencia de alteraciones electromagnéticas.

Finalmente, en la Fig. 18 se presenta la reacción del péndulo ante variaciones de la señal de control.

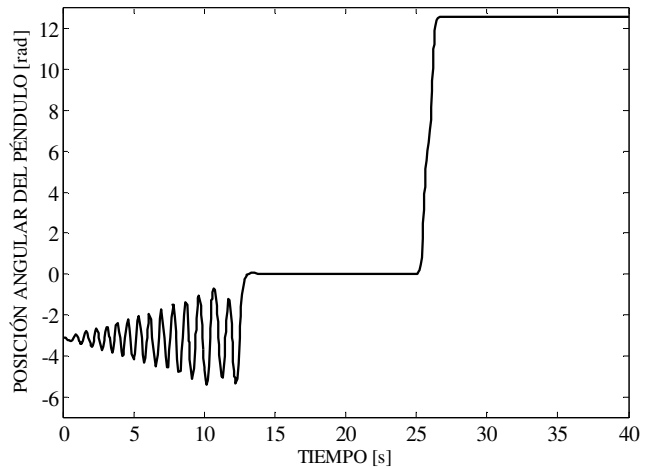

Figura 18. Comportamiento de la posición angular del péndulo ante una operación con perturbaciones en la señal de control

Cuando la señal de control se hace nula entre los $11 \mathrm{~s}$ y $12.5 \mathrm{~s}$ se observa que el péndulo pierde amplitud en la magnitud del balance, por lo cual una vez se reactiva la señal de control, este tarda en alcanzar la posición vertical invertida. Por otro lado, cuando hay una perturbación en la magnitud de la señal de control a los $25 \mathrm{~s}$, el péndulo se sale de la poción de operación y es obligado a dar dos vueltas completas antes de que el controlador REVE sea capaz de estabilizarlo en los $4 \pi \mathrm{rad}$ $\left(720^{\circ}\right)$.

\section{CONCLUSIONES}

Las RNA muestran ser una estrategia eficiente a la hora de aprender y generalizar las tareas de control. Son particularmente útiles para diseñar tareas de control en las que no se cuenta como modelos matemáticos que determinen la dinámica no lineal de planta y en las que sólo se tienen mediciones de señales de entrada y salida; ya que determinan los patrones de comportamiento del sistema y tienen la capacidad de actuar ante fenómenos nunca visto en el conjunto de entrenamiento.

El control por REVE muestra ser un control capaz de ampliar la zona de operación de un sistema no lineal alrededor de su punto de operación, con lo que tiende a generar señales de control lo suficientemente rápidas, para estabilizar las variables de estado y garantizar regulación de las mimas, aunque esto depende claramente de los polos de referencia seleccionados en la etapa de diseño.

Se verifica que utilizar una estrategia de conmutación de tres niveles para sistemas pendulares, reduce la aparición de picos en la señal de control en el momento del intercambio de los controladores, ya que se hace uso de la inercia de la planta para reducir las condiciones iniciales de las variables de estado, emulando un comportamiento cercano al reposo, cuando entra acción el controlador por REVE.

El diseño de controladores por medio de RNA no corresponde a la implementación de una estrategia determinística, y por tanto, requiere en gran medida de la destreza del diseñador, para seleccionar su arquitectura, es decir, el número de capas y neuronas por capa, así como sus funciones de transferencia y el algoritmo más indicado para su entrenamiento. Por lo que se puede afirmar en forma general que el diseño de RNA, es un arte heurístico que requiere de pruebas de ensayo y error para ajustar todos los parámetros que intervienen en su elaboración.

El diseño de las pruebas para verificar la eficiencia de la estrategia de control propuesta, hace parte fundamental de la puesta en funcionamiento de cualquier sistema de control, por lo cual requiere de un estudio detallado de los posibles fenómenos que pueden alterar el normal comportamiento del sistema físico, con el fin de realizar un proceso de retroalimentación que ayude a hacer más robusto al sistema de control y así, para prevenir escenarios de operación indeseados.

\section{AGRADECIMIENTOS}

Agradecimientos especiales a la Universidad Tecnológica de Pereira (UTP) y al Instituto Tecnológico Metropolitano de Medellín (ITM), en especial al grupo de investigación MATYER, por permitir el uso de equipos y tiempo en el desarrollo de este trabajo de investigación.

\section{REFERENCIAS}

[1] Mahmud, K., "Design and analysis of the control of an inverted pendulum system by Matlab," Global High Tech Congress on Electronics (GHTCE), 2013 IEEE, vol., no., pp.207,211, 17-19 Nov. 2013. doi: 10.1109/GHTCE.2013.6767275

[2] Chung-Huang Yu; Fu-Cheng Wang; Yu-Ju Lu, "Robust control of a Furuta pendulum," SICE Annual Conference 2010, Proceedings of, vol., no., pp.2559,2563, 18-21 Aug. 2010.

[3] Wei Li; Tanaka, K.; Wang, H.O., "Acrobatic control of a pendubot," Fuzzy Systems, IEEE Transactions on, vol.12, no.4, pp.549-554, Aug. $2004 . \quad$ doi: 10.1109/TFUZZ.2004.832540 
[4] Ó. O. Rodríguez Díaz, E. L. Téllez Valderrama, and D. A. Gutiérrez Ramírez, "Simulating a Rotational Inverted Pendulum Model by using Matlab and Easy Java Simulations," Tecno Lógicas, no. 28, pp. 15-32, 2012.

[5] Prasad, L.B.; Tyagi, B.; Gupta, H.O., "Modelling and Simulation for Optimal Control of Nonlinear Inverted Pendulum Dynamical System Using PID Controller and LQR," Modelling Symposium (AMS), 2012 Sixth Asia , vol., no., pp.138,143, 29-31 May 2012. doi: 10.1109/AMS.2012.21

[6] O. D. Montoya, J. G. Valenzuela, and D. Giraldo, "Control global del Péndulo Rotacional Invertido empleando modelos de energía," Sci. Tech., vol. XVII, no. 52, pp. 1625, 2012.

[7] R. Iriarte, L. T. Aguilar, and L. Fridman, "Second order sliding mode tracking controller for inertia wheel pendulum," J. Franklin Inst., vol. 350, no. 1, pp. 92-106, Feb. 2013.

[8] J. G. Hoyos, J. E. Cardona, and R. Arango, "Control en línea con algoritmos genéticos y recocido simulado," Sci. Tech., vol. XIII, no. 35, pp. 113-116, 2007.

[9] M. A. Martínez, J. Sanchis, and X. Blasco, "Algoritmos Genéticos Aplicados al Diseño de Controladores Robustos," Rev. Iberoam. automática e informática Ind. ( RIAI ), vol. 3, no. 1, pp. 39-51, 2006.

[10]Baojiang Zhao; Shiyong Li, "Design of a Fuzzy Logic Controller by Ant Colony Algorithm with Application to an Inverted Pendulum System," Systems, Man and Cybernetics, 2006. SMC '06. IEEE International Conference on, vol.5, no., pp.3790-3794, 8-11 Oct. 2006. doi: 10.1109/ICSMC.2006.384720

[11] Shaoqiang Yuan; Dong Wang; Xingshan Li, "The Application of PSO Algorithm on PenduBot Control," Natural Computation, 2009. ICNC '09. Fifth International Conference on, vol.3, no., pp.329-333, 14-16 Aug. 2009. doi: 10.1109/ICNC.2009.531

[12] L. F. Escobar Dávila, O. D. Montoya Giraldo, and D. Giraldo Buitrago, "Control Global del Péndulo de Furuta Empleando Redes Neuronales Artificiales y Realimentación de Variables de Estado," Tecno Lógicas, no. 30, pp. 71-94, 2013.

[13] J. Wu, H. Su, and T. Wu, "ANN Control of Inverted Pendulum," in 2008 First International Conference on Intelligent Networks and Intelligent Systems, 2008, pp. 912.

[14] Jian-Xin Xu; Zhao-Qin Guo; Tong Heng Lee, "An optimal fuzzy logic controller for an underactuated unicycle," IECON 2011 - 37th Annual Conference on IEEE Industrial Electronics Society, vol., no., pp.2335-2340, 7-10 Nov. 2011. doi: 10.1109/IECON.2011.6119674

[15] M. W. Spong, P. Corke, and R. Lozano, "Nonlinear control of the Reaction Wheel Pendulum," Automatica, vol. 37, no. 11, pp. 1845-1851, Nov. 2001.

[16] Bapiraju, B.; Srinivas, K.N.; Prem Kumar, P.; Behera, L., "On balancing control strategies for a reaction wheel pendulum," India Annual Conference, 2004. Proceedings of the IEEE INDICON 2004. First, vol., no., pp.199-204, 20-22 Dec. 2004. doi: 10.1109/INDICO.2004.1497738

[17] O. D. Montoya Giraldo, L. F. Grisales Noreña, V. D. Correa Ramírez, and D. Giraldo Buitrago, "Control global del péndulo con rueda de reacción mediante regulación de energía y linealización extendida de las variables de estado," Tecno Lógicas, no. 32, pp. 33-46, 2014.

[18]F. Jepsen, A. Soborg, A. R. Pedersen, and Z. Yang, "Development and control of an inverted pendulum driven by a reaction wheel," in 2009 International Conference on Mechatronics and Automation, 2009, pp. 2829-2834.

[19] A. A. Bobtsov, A. A. Pyrkin, and S. A. Kolyubin, "Adaptive stabilization of a reaction wheel pendulum on moving LEGO platform," in 2009 IEEE International Conference on Control Applications, 2009, pp. 1218-1223.

[20] B. R. Andrievsky, "Global stabilization of the unstable Reaction-Wheel Pendulum," Autom. Remote Control, vol. 72, no. 9, pp. 1981-1993, Sep. 2011.

[21] O. D. Montoya, J. G. Valenzuela, and D. Giraldo, "Lógica Difusa Aplicada al Control Local del Péndulo Invertido con Rueda de Reacción," Sci. Tech., vol. 18, no. 4, pp. 623$632,2013$.

[22] D. Giraldo, V. D. Correa, and A. Molina, "Control global para el péndulo con rueda de reacción," Sci. Tech., vol. XIII, no. 35, pp. 95-99, 2007.

[23] Muskinja, N.; Tovornik, B., "Swinging up and stabilization of a real inverted pendulum," Industrial Electronics, IEEE Transactions on, vol.53, no.2, pp.631639, April 2006. doi: 10.1109/TIE.2006.870667

[24] Iwashiro, M.; Furuta, K.; Astrom, K.J., "Energy based control of pendulum," Control Applications, 1996., Proceedings of the 1996 IEEE International Conference on, vol., no., pp.715-720, 15-18 Sep 1996. doi: 10.1109/CCA.1996.558953

[25] C. Alberto de Albuquerque Silva, A. Duarte Doria Neto, J. Alberto Nicolau Oliveira, J. Dantas Melo, D. Simonetti Barbalho and A. Medeiros Avelino, "Definition of an Architecture to Configure Artificial Neural Networks Topologies Using Partial Reconfiguraton in FPGA," in IEEE Latin America Transactions, vol. 13, no. 7, pp. 20942100, July 2015.

[26] J. C. Vaschetti and V. H. Sauchelli, "Artificial Neural Network Applied to The Problem of Secondary Voltage Control," in IEEE Latin America Transactions, vol. 10, no. 2, pp. 1518-1524, March 2012. doi: 10.1109/TLA.2012.6187595 